\title{
Macromolecular crowding effects on reactions of TePixD (Tll0078).
}

\section{AUTHOR(S):}

Toyooka, Tsuguyoshi; Tanaka, Keisuke; Okajima, Koji; Ikeuchi, Masahiko; Tokutomi, Satoru; Terazima, Masahide

\section{CITATION:}

Toyooka, Tsuguyoshi ...[et al]. Macromolecular crowding effects on reactions of TePixD (Tll0078).. Photochemistry and photobiology 2010, 87(3): 584-589

\section{ISSUE DATE:}

2010-11-29

URL:

http://hdl.handle.net/2433/197221

\section{RIGHT:}

This is the peer reviewed version of the following article: Toyooka, T., Tanaka, K., Okaiima, K., Ikeuchi, M., Tokutomi, S. and Terazima, M. (2011), Macromolecular Crowding Effects on Reactions of TePixD (TIl0078). Photochemistry and Photobiology, 87: 584-589, which has been published in final form at http://dx.doi.org/10.1111/j.1751-

1097.2010.00849.x:この論文は出版社版でありません。引用の際には出版社版をご確認ご利用ください。; This is not the published version. Please cite only the published version. 


\section{Macromolecular Crowding Effects on Reactions of TePixD (Tll0078)}

Tsuguyoshi Toyooka ${ }^{1}$, Keisuke Tanaka ${ }^{1}$, Koji Okajima ${ }^{2,3}$, Masahiko Ikeuchi ${ }^{2}$, Satoru Tokutomi $^{3}$, Masahide Terazima ${ }^{{ }^{*}}$

${ }^{1}$ Department of Chemistry, Graduate School of Science, Kyoto University, Kyoto 606-8502, Japan

${ }^{2}$ Department of Life Sciences (Biology), Graduate School of Arts and Sciences, The University of Tokyo, Meguro, Tokyo 153-8902, Japan

${ }^{3}$ Research Institute for Advanced Science and Technology, Department of Biological Science, Graduate School of Science, Osaka Prefecture University, Sakai, Osaka 599-8531, Japan

* Corresponding author

mterazima@kuchem.kyoto-u.ac.jp 


\begin{abstract}
To reveal macromolecular crowding effects on a chemical reaction of a BLUF (sensors of blue light using FAD) protein (PixD from a thermophilic cyanobacterium Thermosynechococcus elongatus BP-1 (TePixD, Tl10078)), the photoreaction was studied at various concentrations of the macromolecule Ficoll-70 by UV/vis absorption spectroscopy and the pulsed laser-induced transient grating (TG) method. The absorption spectrum did not change with varying concentration of Ficoll-70. The crowding did not affect the quantum yield of the spectral red shift reaction, recovery rate of the product, rate constant of the volume change reaction, and the magnitude of the volume change. However, the magnitude of the TG signal representing the diffusion sensitive conformation change significantly increased on addition of Ficoll-70. This dependence was attributed to the crowding effect on the TePixD decamer-tetramer equilibrium in the solution. This result indicates that the TePixD reaction is more efficient in cellular than in in vitro conditions.
\end{abstract}




\section{Introduction}

A characteristic of the interiors of living cells is high concentrations of macromolecules [1-4], which is referred to as 'crowding conditions'. Under such conditions chemical reactions may differ from those occurring in dilute solution. Crowding influences various molecular as well as biological properties [5]. Understanding how the crowding environment affects a biological reaction should be important in biophysical studies. For example, an extensive study of the macromolecular crowding effect was performed for a protein folding reaction, e.g., by Stagg et al., who reported that folded flavodoxin is stabilized compared with the unfolded state in a crowded environment [6,7]. The crowding effects on aggregation, association and dissociation of proteins have also been studied [8-12].

In contrast to these thorough studies on static characteristics of protein conformations, crowding effects on chemical reactions that are related to biological function have not been well investigated. In the present study we investigated the photochemical reaction of PixD from a thermophilic cyanobacterium Thermosynechococcus elongatus BP-1 (TePixD, Tl10078) in a crowded environment, to reveal how the reaction is affected by the presence of macromolecules. TePixD is a $17 \mathrm{kDa}$ protein that consists of the BLUF (sensors of blue light using FAD) domain and short helices $[13,14]$. The biological function of PixD protein is considered to control the phototaxis movement on the basis of research of a mesophilic cyanobacterium Synechocystis (SyPixD, Slr1694). The reported crystal structure of TePixD showed two pentamer rings that form a decamer $[15,16]$. The decameric structure is considered to reflect the biological activity, since TePixD maintains an oligomeric structure in solution and previous SyPixD studies showed that light illumination controls the oligomeric states and also the intermolecular interaction with the transducer protein $[16,17]$. Studies by absorption spectroscopy of the photochemical reaction of TePixD revealed that this protein exhibits typical photochemistry of the BLUF proteins. The absorption spectrum is red shifted (red-shifted intermediate) upon photoexcitation within a 10 ns-excitation pulse width [18-20]. Although the spectrum does not change after this initial reaction, and reverts to the dark state with a time constant of $12 \mathrm{~s}$ at room temperature, two spectrally silent phases were observed: the partial molar volume change with a time constant of $40 \mu \mathrm{s}$, and a diffusion coefficient $(D)$ change (i.e. a diffusion-sensitive conformational change) with a time constant of $4 \mathrm{~ms}$ [20]. The previous studies were conducted in dilute solution, and it was of interest to study the macromolecular crowding effect on this reaction to obtain information on the reaction in vivo.

To mimic the interior of living cells, we investigated the TePixD reaction in a buffer solution with high macromolecular content. We selected Ficoll-70 as macromolecular background species, since it represents an inert, highly crosslinked sucrose polymer. Ficoll-70 has molecular mass $\sim 70 \mathrm{kDa}$ and hydrodynamic radius $5.1-5.5 \mathrm{~nm}$, and has been widely used in experimental crowding studies.[1,6-8, 21-25]

In the present study, the crowding effect was investigated by UV/vis absorption spectroscopy and the pulsed laser induced transient grating (TG) method. The absorption method 
is sensitive to conformation change around the chromophore (FAD), whereas the TG signal intensity and the temporal profile reflect the global conformation change including change far from the chromophore and the intermolecular interaction. We found that the crowding effect on the absorption spectrum of the ground state species, recovery kinetics of the product, and absorption change induced by light are almost negligible indicating that the conformation, at least around the chromophore is rather stable against the presence of the macromolecules. On the other hand, a significant increase of the TG signal representing the diffusion sensitive conformation change was observed. This result is explained by the crowding effect on the aggregation state of TePixD.

\section{Materials and Methods}

\section{Experimental}

Sample preparation

TePixD was expressed using a pET28a vector transformed into E. coli BL21(DE3) and purified by nickel affinity column chromatography, as reported previously [15]. All measurements were performed in Hepes buffer (elution buffer: $12 \mathrm{mM}$ Hepes- $\mathrm{NaOH}(\mathrm{pH} 7.5)$, $500 \mathrm{mM} \mathrm{NaCl}, 500 \mathrm{nM}$ imidazole). Ficoll-70 (Sigma-Aldrich) was used for the crowding reagent. The concentration of TePixD was determined by absorption measurement. The absorbance at 465 $\mathrm{nm}$ was adjusted to ca. 0.5 , and slight variation in the concentration was corrected using the absorbance at $465 \mathrm{~nm}$.

\section{Measurements}

The experimental setup for the TG measurement was similar to that reported previously $[26,27]$. Briefly, a laser pulse from a dye laser (Lumomics, HyperDye 300; wavelength=465 nm) pumped by an excimer laser (Lambda Physik, $\mathrm{XeCl}$ operation; $308 \mathrm{~nm}$ ) was used as an excitation beam, and a diode laser $(835 \mathrm{~nm})$ as probe beam. The excitation beam was split into two by a beam splitter, and crossed inside the sample cell. The TG signal was isolated from the excitation laser beam with a glass filter and a pinhole, and detected by a photomultiplier tube (Hamamatsu R1477). The signal was recorded with a digital oscilloscope (TDS-7104; Tektronix). The spacing of the fringes was measured by the decay rate constant of the thermal grating signal from a calorimetric reference sample (bromocresol purple), which releases all of the photon energy of the excitation as thermal energy within the time response of our system. To avoid possible multi-excitations, the repetition rate of the excitation was $0.03 \mathrm{~Hz}$.

In the transient absorption measurement, the same pumped laser light as that for TG measurement was used. An Ar ion laser (Coherent INNOVA 300; $\lambda=477 \mathrm{~nm}$ ) was used for the probe light, which was introduced into the sample by counter-propagation geometry. The probe light was detected by the photomultiplier tube, and the time profile of the signal was recorded by the digital oscilloscope.

Viscosities of solutions were measured with an automated Micro Viscometer (Anton Par 
AMVa).

All samples were filtered with a centrifugal filter (10000 M.W.) before use.

\section{Results and discussion}

\section{Crowding effect on conformation and reaction around the chromophore}

Before studying the crowding effect on the reaction, the crowding effect on the initial conformation was examined by UV/vis absorption spectroscopy. The absorption spectrum reflects the conformation around the chromophore. Figure 1 depicts the absorption spectra of TePixD at various concentrations of Ficoll-70 (0-300 $\left.\mathrm{g} \mathrm{L}^{-1}\right)$. The shape of the absorption spectrum did not depend on the concentration of the crowding reagent indicating that the crowding effect on the dark state conformation, at least around the chromophore is negligible.

We next investigated the reaction of TePixD monitored by the pulsed laser induced absorption change. After excitation at $465 \mathrm{~nm}$, the absorption at $477 \mathrm{~nm}$ increased by the creation of the red-shifted species. The signal returned to the baseline by the dark reversion reaction. The amplitude of the change and the recovery kinetics were independent of the concentration of Ficoll (0-300 $\left.\mathrm{g} \mathrm{L}^{-1}\right)$ within experimental error (Fig. 2). The negligible crowding effect on the amplitude (Fig. 2(B)) indicates that the reaction yield (at least as monitored by the absorption change) does not depend on the concentration of Ficoll-70. Furthermore, the recovery rate was also insensitive to the concentration of Ficoll-70 (Fig. 2(C)).

These negligible crowding effects on the dark state conformation, reaction yield, and recovery kinetics suggest that the chromophore is well protected from the macromolecules in the environment, and the conformation around the chromophore is insensitive to the inert macromolecules in the solution.

\section{Crowding effect on spectrally silent reaction}

For a large system such as proteins, conformation changes far from a chromophore could be different from that around the chromophore. The TG method is a powerful technique for detecting spectrally silent dynamics [16,26,28]. The TG signals of TePixD with and without Ficoll-70 are depicted in Fig.3. The TG signal observed after photoexcitation of TePixD without Ficoll-70 was analyzed previously [19]. The TG signal intensity is proportional to the square of the photo-induced refractive index change modulation $(\delta n)[19,26,27,29]$. The signal consists of $\delta$ n due to the temperature change $\left(\delta n_{\mathrm{th}}(\mathrm{t})\right.$; thermal grating) and that of the created (or depleted) chemical species (the species grating). The species grating signal intensity is given by the difference of the refractive index changes due to the reactant $\left(\delta n_{R}\right)$ and product $\left(\delta n_{P}\right)$. The total TG signal $\left(I_{T G}(t)\right)$ is expressed as:

$$
I_{T G}(t)=\alpha\left\{\delta n_{t h}(t)+\delta n_{v} \exp \left(-t / \tau_{v}\right)+\delta n_{P}(t)-\delta n_{R}(t)\right\}^{2}
$$

where $\alpha$ is a constant, $\delta n_{v}$ is the refractive index change due to the volume change (spectrally silent dynamics), and $\tau_{\mathrm{v}}$ is the time constant of the volume change. In a sufficiently long time 
region, the molecular diffusion coefficient $(D)$ is time independent and the temporal profile of the species grating signal decays with a rate constant of $D q^{2}$ for the reactant and the product. Hence, the time development of the TG signal for describing the molecular diffusion part can be expressed by: [19,26,29]

$$
I_{T G}(t)=\alpha\left\{\delta n_{t h}(t)+\delta n_{v} \exp \left(-t / \tau_{\mathrm{v}}\right)+\delta n_{P} \exp \left(-D_{P} q^{2} t\right)-\delta n_{R} \exp \left(-D_{R} q^{2} t\right)\right\}^{2}
$$

where $D_{\mathrm{R}}$ and $D_{\mathrm{P}}$ are diffusion coefficients of the reactant and the product, respectively. Furthermore, $\delta n_{R}(>0)$ and $\delta n_{P}(>0)$ are, respectively, the initial refractive index changes due to the changes in the reactant and the product concentrations by the reaction.

When Ficoll-70 was added to the solution, we found that both the amplitude and the rate of the volume change did not depend on the concentration of the macromolecule (Fig.3(B)). The amplitude of this component reflects the reaction yield and the volume change $(\Delta \mathrm{V})$. Considering the concentration independence of the reaction yield monitored by the transient absorption method, we concluded that the magnitude of $\Delta \mathrm{V}$ is also independent of the presence of Ficoll-70. It is interesting to find that not only the amplitude but also the kinetics of $\Delta \mathrm{V}$ did not depend on the concentration of Ficoll-70, although the viscosity of the solution increased significantly by adding Ficoll-70. The insensitivity of the kinetics to the viscosity of the medium suggests that this volume change reflects a local change in the conformation, not large amplitude motion.

The most significant crowding effect was observed for the diffusion signal. The peak of the diffusion signal shifted to longer time range and the intensity increased with increasing concentration (Fig.4(A)). The temporal shift by adding Ficoll-70 is reasonable, because the solution viscosity increases and diffusion should be affected by the viscosity. According to the Stokes-Einstein equation, the translational diffusion coefficient in solution is described by $[6,30]$

$$
\mathrm{D}=\mathrm{kB} \mathrm{T} / 6 \pi \eta \mathrm{r}
$$

where $r$ is the hydrodynamic radius of the molecule approximated as a sphere, $\mathrm{T}$ is the temperature, and $\eta$ is the viscosity of the solvent ( $k_{B}$ is the Boltzmann constant). We determined $D_{P}$ and $D_{R}$ from the TG signal at low $\mathrm{q}^{2}$ and found that these diffusion coefficients are proportional to $\eta^{-1}$ (Fig.4(B)).

The increase of the diffusion peak intensity is rather surprising, because as mentioned above the reaction yield did not depend on the concentration of Ficoll-70. The origin of this crowding effect is discussed in the next section.

\section{Origin of the crowding effect}

As described above, the reaction quantum yield of the spectral red shifted species and the kinetics did not depend on the crowding, but the signal intensity reflecting the diffusion sensitive conformation change was crowding-dependent. The only possible explanation of these two facts is that all of the initial spectral red shifted species does not induce the conformation change. Previously, we found that TePixD in solution in the dark is in equilibrium between a reactive species exhibiting the diffusion-sensitive conformational change and a non-reactive 
species [19]. Since it was found that the relative proportions of these species were dependent on the concentration, the difference in the reactivity was attributed to the different oligomeric states of TePixD, i.e. pentamer and decamer. These results demonstrated that only the decamer state is responsible for the conformational change. Considering this fact, we suggest that crowding affects the decamer-pentamer equilibrium; that is, the decamer contribution increased with increasing concentration of Ficoll-70. Since the diffusion signal intensity was saturated at $\left[\right.$ Ficoll-70] $=200 \mathrm{~g} \mathrm{~L}^{-1}$, we can assume that all PixD is in the decamer state at this concentration. Therefore, the fraction of the decamer ( $\mathrm{f}($ decamer $)$ ) may be calculated from the diffusion signal intensity, and Figure 4 shows the fraction of the decamer versus the concentration of Ficoll-70.

Macromolecular crowding is expected to have several significant effects on protein aggregation, the major effects being those due to excluded volume and increased viscosity. To examine the viscosity effect we investigated the effect of adding glycerol, which is capable of increasing the solution viscosity. Figure 5 shows the TG signals at various concentrations of glycerol. The viscosity of $300 \mathrm{~g} \mathrm{~L}^{-1}$ Ficoll-70(14 cP) is comparable with that of $810 \mathrm{~g} \mathrm{~L}^{-1}$ glycerol (Fig.6). Interestingly, the intensity of the diffusion peak decreased on addition of glycerol, suggesting that the decamer dissociates in the presence of glycerol. Furthermore, it is hard to consider that viscosity change influences the fraction of the oligomeric states. Hence, we concluded that the crowding effect of TePixD oligomer structure is due to the volume effect of the macromolecule.

Both steric repulsion and enthalpic contributions may contribute to the crowding effect. To elucidate the crowding effects, specific or non-specific binding interactions between TePixD and crowding agent (the enthalpic contributions) are not desirable. It is important to ensure that TePixD does not interact with the background macromolecule (Ficoll-70), except by steric repulsion. Steric repulsion is caused by the impenetrability of solute molecules and is ubiquitously present in crowded environments. On the other hand, specific or non-specific binding interactions between TePixD and crowding agent (the enthalpic contributions) have to be excluded. To address the problem of binding interactions of Ficoll-70 with TePixD, we examined the diffusion of TePixD under the crowding conditions compared with dilute solutions. Since the hydrodynamic radius of Ficoll-70 is relatively large, any binding interaction between TePixD and Ficoll-70 would have been detected by longer diffusion times than expected from the viscosity change. However, the change in D was reasonably explained by the viscosity change, as noted above. Hence we excluded the enthalpic contribution to the observed crowding effect.

In aqueous solution, Ficoll-70 macromolecules occupy a considerable volume fraction that is inaccessible to TePixD. The observed crowding effect may be explained by the excluded volume effect. The excluded volume effect can be described as an effective attractive interaction between two spheres in a polymer solution, which is induced by the inability of the polymer molecules to enter the volume between the two spheres, when their separation distance is smaller than the size of the polymer molecule [6,8]. It is reasonable to consider that, because of this effective attractive interaction, the pentamer of TePixD tends to convert to the decamer state, 
which is reactive.

Since macromolecular crowding is an intrinsic feature of the cellular environment, there has been considerable recent interest in this effect. However, as far as we aware, there has been no investigation of the crowding effect on the photo-reactivity of biological proteins. This study is the first example showing that the reaction yield of a photosensor protein can be enhanced in cellular conditions compared with the yield in dilute solution.

In summary, the macromolecular crowding effect on the chemical reaction of TePixD was studied. The absorption spectrum of the ground state did not depend on the crowding agent, indicating that the conformation is not sensitive to crowding. Similarly, we did not detect any crowding effect on the reaction quantum yield of the spectral red shifted species, dark recovery dynamics, and conformation change detected by the volume change with a time constant of 40 $\mu \mathrm{s}$. On the other hand, the conformation change that changes the diffusion coefficient increased significantly in the presence of Ficoll-70. This effect is explicable in terms of the excluded volume effect increasing the proportion of TePixD decamer in the crowded condition. This result suggests that the light sensitivity of TePixD is much larger in cells than in dilute solution.

\section{Acknowledgments}

This work was supported by grants-in-aid from the MEXT Japan to M.T. (No. 15076204, 18205002), S.T. (No.17084008) and from the JSPS Japan to S.T. (No.19370070). 


\section{Reference}

1. Ellis, R. J. (2001) Macromolecular crowding:obvious but underappreciated. Trends Biochem. Sci. 26, 597-604.

2. Minton, A. P. (2000). Implications of macromolecular crowding for protein assembly. Curr. Opin. Struct. Biol. 10, 34-39.

3. Minton, A. P. (2000). Effect of a concentrated 'inert' macromolecular cosolute on the stability of a globular protein with respect to denaturation by heat and by chaotropes: a statistical-thermodynamic model. Biophys. J. 78 101-109.

4. Minton A. P. (2001). The influence of macromolecular crowding and macromolecular confinement on biochemical reactions in physiological media. J. Biol. Chem. 276, 10577-10580.

5. Felix, R., H. Marcus, S. Tilo, Z. Fajun, W.A. S. Maximilian, Z. Stefan, Robert M.J. J., M. Marco, F. Peter and S. Frank (2010). Protein diffusion in crowded electrolyte solutions. Biochim Biophys Acta 1804, 68-75.

6. Noga, K. and S. Gideon (2004). Effect of Crowding on Protein-Protein Association Rates: Fundamental Differences between Low and High Mass Crowding Agents. J. Mol. Biol. 336, 763-774.

7. Michael, P., S. Loren and W. Pernilla (2007). Macromolecular crowding increases structural content of folded proteins. FEBS Lett. 581, 5065-5069.

8. Noga, K., Y. K. Yosef, H. Gilad and S. Gideon (2007). Protein-Protein Association in Polymer Solutions: From Dilute to Semidilute to Concentrated. Biophy. J. 92, 2139-2149

9. Yu-Ling, Z., L. Jun-Ming, C. Jie and L. Yi (2007). Molecular crowding enhances native structure and stability of $\alpha / \beta$ protein flavodoxin, Proc Natl Acad Sci U S A 104, 18976-18981.

10. Rosgen, J., M. Pettitt and D. W. Bolen (2005). Protein Folding, Stability, and Solvation Structure in Osmolyte Solutions. Biophys. J. 89, 2988-2997.

11. Cheung, M. S. and D. Thirumalai (2007). Effects of Crowding and Confinement on the Structures of the Transition State Ensemble in Proteins. J. Phys. Chem. B 111, 8250.

12. Sasahara, K., P. McPhie and A. P. Minton (2003). Effect of dextran on protein stability and conformation attributed to macromolecular crowding. J. Mol. Biol. 326, 1227-1237.

13. Kita, A., K. Okajima, Y. Morimoto, M. Ikeuchi and K. Miki (2005). Structure of a Cyanobacterial BLUF Protein, T110078, Containing a Novel FAD-binding Blue Light Sensor Domain. J. Mol. Biol. 349, 1-9.

14. Hua, Y., A. Spencer, M. Shinji, D. Vladimira, M. Keith and B. Carl (2006). Crystal Structures of the AppA BLUF Domain Photoreceptor Provide Insights into Blue Light-mediated Signal Transduction. J. Mol. Biol. 362, 717-732.

15. Okajima, K., S. Yoshihara, Y. Fukushima, G. Xiaoxing, M. Katayama, S. Higashi, M. Watanabe, S. Sato, S. Tabata, Y. Shibata, S. Itoh and M. Ikeuchi (2005). Biochemical and Functional Characterization of BLUF-Type Flavin-Binding Proteins of Two Species of 
Cyanobacteria. J. Biochem. 137, 741-750.

16. Ziraka, P., A. Penzkofera, C. Lehmpfuhlb, T. Mathesb and P. Hegemannb (2007).

Absorption and emission spectroscopic characterization of blue-light receptor Slr1694 from Synechocystis sp. PCC6803. J. Photochem. Photobiol. B 86, 22-34.

17. Astrid, J., R. Jochen, D. Tatiana, L. S. Robert and S. Ilme (2006) Crystal Structures of the Synechocystis Photoreceptor Slr1694 Reveal Distinct Structural States Related to Signaling. Biochemistry 45, 12687-12694.

18. Masuda, S., K. Hasegawa and T. A. Ono (2005). Tryptophan at position 104 is involved in transforming light signal into changes of beta-sheet structure for the signaling state in the BLUF domain of AppA. Plant Cell Physiol. 46, 1894-1901.

19. Okajima, K., Y. Fukushima, H. Suzuki, A. Kita, Y. Ochiai, M. Katayama, Y. Sshibata, K. Miki, T. Noguchi, S., Itoh and M., Ikeuchi (2006). Fate Determination of the Flavin Photoreceptions in the Cyanobacterial Blue Light Receptor TePixD (T110078). J. Mol. Biol. 363, 10-18.

20. Tanaka K., Y. Nakasone, K. Okajima, M. Ikeuchi, S. Tokutomi and M. Terazima (2009). Oligomeric-State-Dependent Conformational Change of the BLUF Protein TePixD (T110078). J. Mol. Biol. 386, 1290-1300.

21. Dirar, H., P. Michael, S. Antonios, S. C. Margaret and W. Pernilla (2008). Crowded, cell-like environment induces shape changes in aspherical protein. Proc Natl Acad Sci U S A 105, 11754-11759.

22. Sanbo, Q. and Z. Huan-Xiang (2009). Atomistic Modeling of Macromolecular Crowding Predicts Modest Increases in Protein Folding and Binding Stability. Biophys. J. 97, 12-19. 23. Yael P., S. Eilon, H. Gilad and S. Gideon (2009). Common Crowding Agents Have Only a Small Effect on Protein-Protein Interactions. Biophys. J. 97, 875-885.

24. Yu-Ling, Z., L. Jun-Ming, C. Jie and L. Yi (2006). Macromolecular crowding enhances the binding of superoxide dismutase to xanthine oxidase: Implications for protein-protein interactions in intracellular environments. Int. J. Biochem. Cell Biol. 38, 1986-1994.

25. Ellis, R J. (2001). Macromolecular crowding: an important but neglected aspect of the intracellular environment. Curr. Opin. Struct. Biol. 11, 114-119.

26. Eitoku, T., Y. Nakasone, D. Matsuoka, S. Tokutomi and M. Terazima (2005). Conformational dynamics of phototropin 2 LOV2 domain with the linker upon photoexcitation. J. Am. Chem. Soc. 127, 13238-13244.

27. Terazima, M. (2006). Diffusion coefficients as a monitor of reaction kinetics of biological molecules. Phys. Chem. Chem. Phys. 8, 545-557.

28. Nakasone, Y., T. Eitoku, D. Matsuoka, S. Tokutomi and M. Terazima (2007). Dynamics of Conformational Changes of Arabidopsis Phototropin 1 LOV2 with the Linker Domain. $J$. Mol. Biol. 367, 432-442.

29. Nishida, S., T. Nada and M. Terazima. (2004). Kinetics of intermolecular interaction during protein folding of reduced cytochrome c. Biophys. J. 87, 2663-2675. 
30. Hannon, L., G. C. Lie and E. Clementi (1986). Molecular dynamics simulation of flow past a plaste. J. Sci. Comput. 1, 145-150. 


\section{Figure captions}

Fig.1 Absorption spectra of TePixD at Ficoll-70 concentrations 0 (red), 50 (orange) 100 (green) 150 (blue) 200 (purple) $\mathrm{g} \mathrm{L}^{-1}$. They are completely overlapped.

Fig.2 (A) Dependence of the transient absorption decay profile on Ficoll-70 concentration. [Ficoll-70] $=0$ (red), 50 (orange) 100 (green) 150 (blue) 200 (purple) g L ${ }^{-1}$. (B) Dependence of the amplitude of the transient absorption change at $\mathrm{t}=0$, which is equivalent to the quantum yield of the formation of the red-shifted species on Ficoll-70 concentration. (C) Dependence of the recovery time constant of the red-shifted species on Ficoll-70 concentration.

Fig.3 (A) Observed TG signals of TePixD measured at [Ficoll-70] $=0$ (broken line) and 200 (solid line) $\mathrm{g} \mathrm{L}^{-1}$ with $q^{2}=7.12 \times 10^{12} \mathrm{~m}^{-2}$. Inset: Amplified TG signal without Ficoll-70 in a short time region. (B) TG signals representing the volume change at various Ficoll-70 concentrations. $\left[\right.$ Ficoll-70] $=0$ (red), 50 (orange) 100 (green) 150 (blue) 200 (purple) $\mathrm{g} \mathrm{L}^{-1}$. The almost completely overlapped curves indicate that the magnitude of the volume change as well as the time constant of this conformation change are independent of the Ficoll-70 concentration.

Fig.4 (A) Diffusion signals of TePixD measured at various Ficoll-70 concentrations with $q^{2}=1.35 \times 10^{11} \mathrm{~m}^{-2}$ : (1) [Ficoll] $=0$, (2) 50, (3) 100, (4)150, (5) $200 \mathrm{~g} \mathrm{~L}^{-1}$. (B) Plots of the diffusion coefficients of the reactant (circles) and the product (squares) against the inverse of the solution viscosity. The best fit lines by the Stokes-Einstein relationship are shown by the linear lines. (C) Fraction of the decamer (f(decamer)) at various concentrations of Ficoll-70.

Fig.5 (A) Observed TG signals of TePixD measured at various glycerol concentrations with $q^{2}=1.32 \times 10^{11} \mathrm{~m}^{-2}$ : the concentrations are (1) 0 , (2) 63 , (3) 126 , (4) 252 , (5) $378 \mathrm{~g} \mathrm{~L}^{-1}$.

Fig.6 Plots of the solution viscosity as a function of the concentration of Ficoll-70 (circles) and glycerol (squares). 


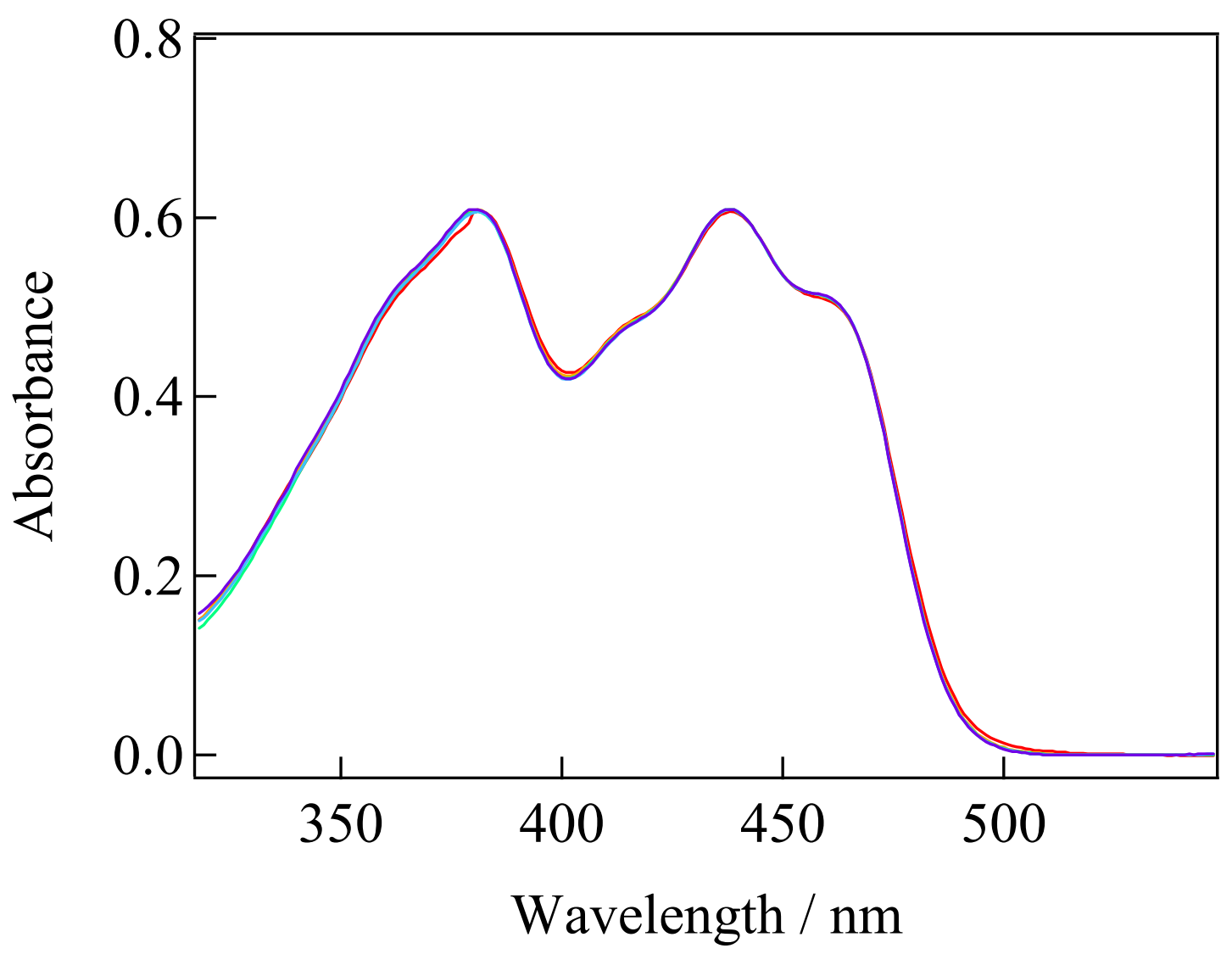

Fig.1 

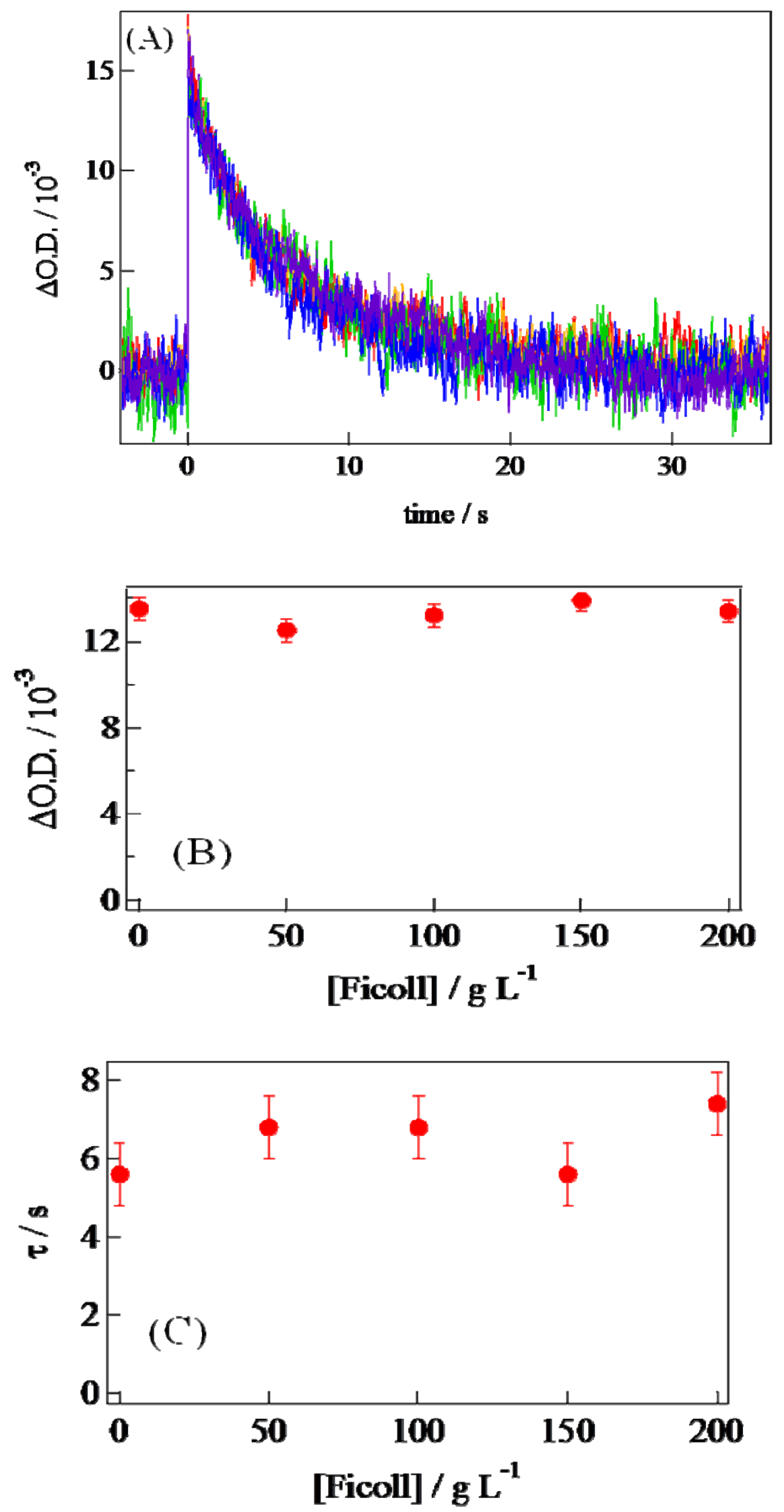

Fig.2 

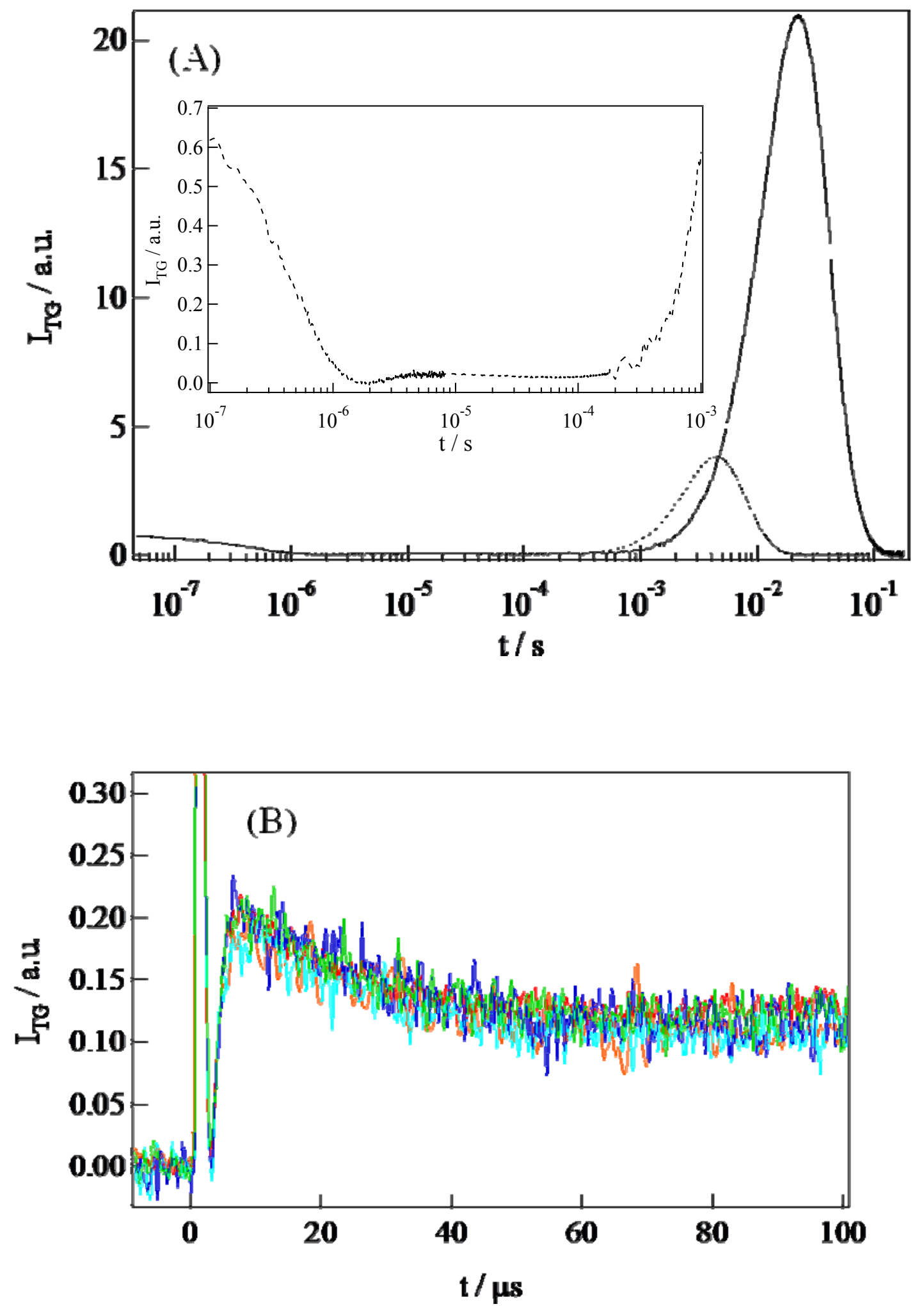

Fig.3 

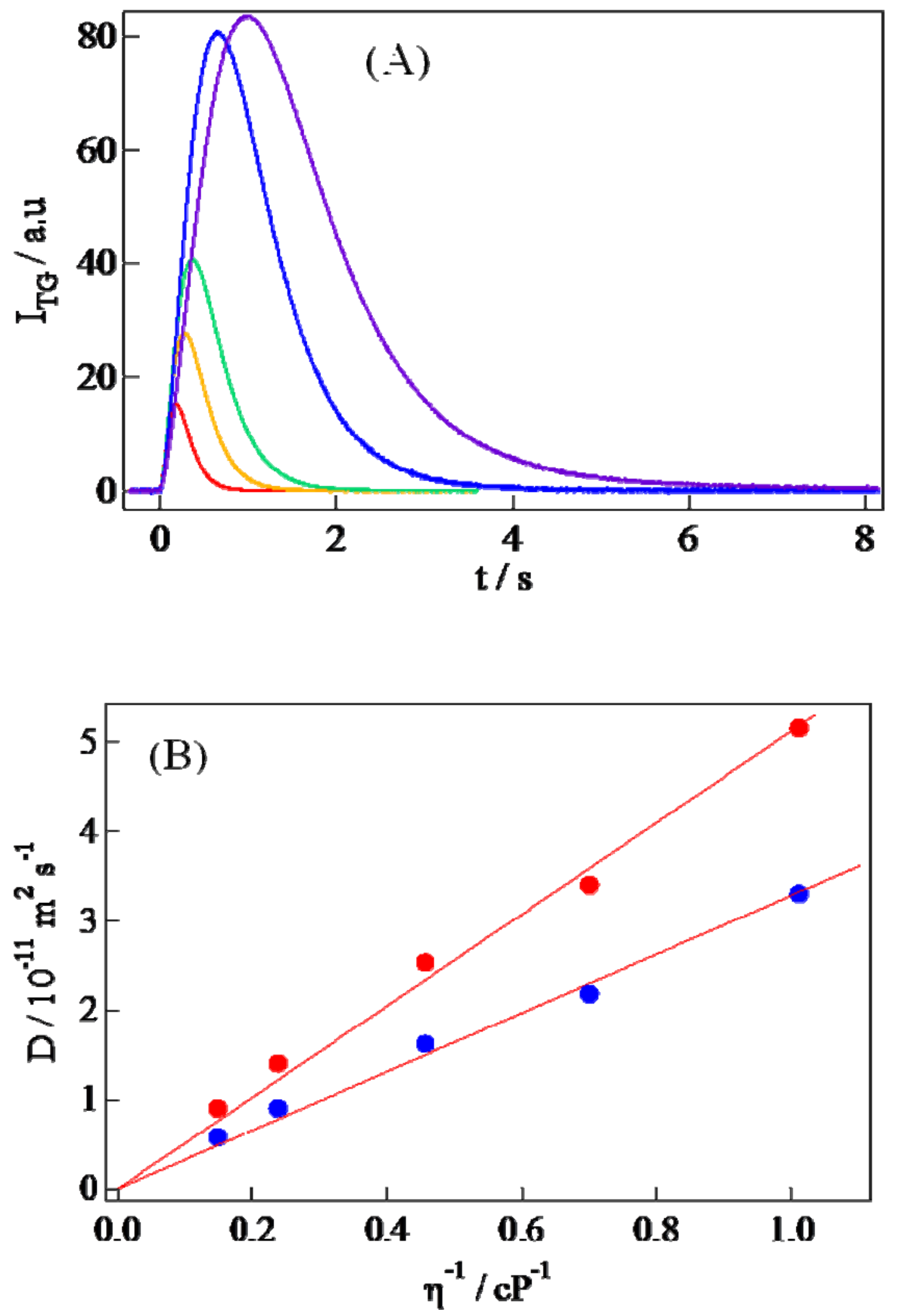


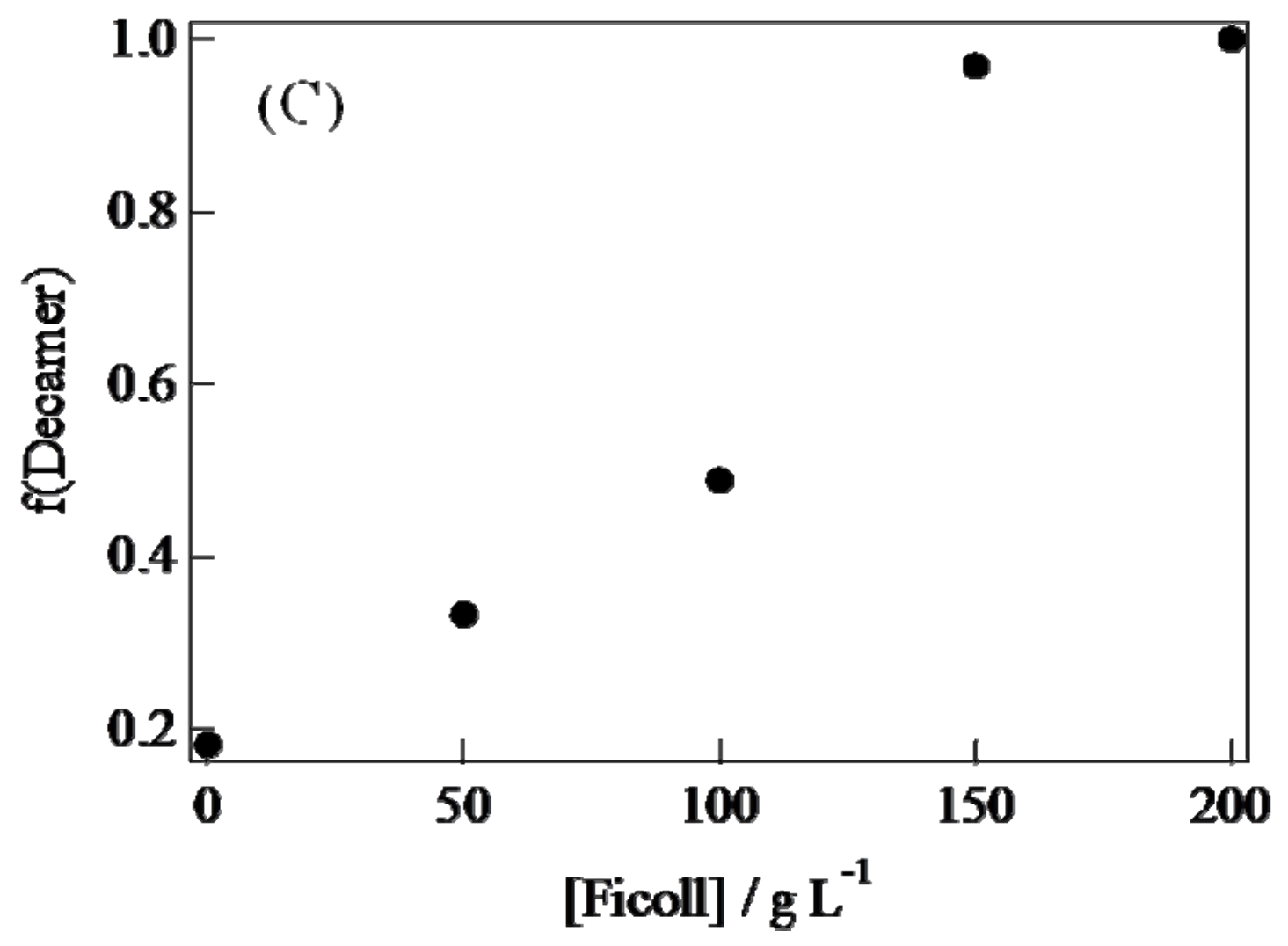

Fig.4 


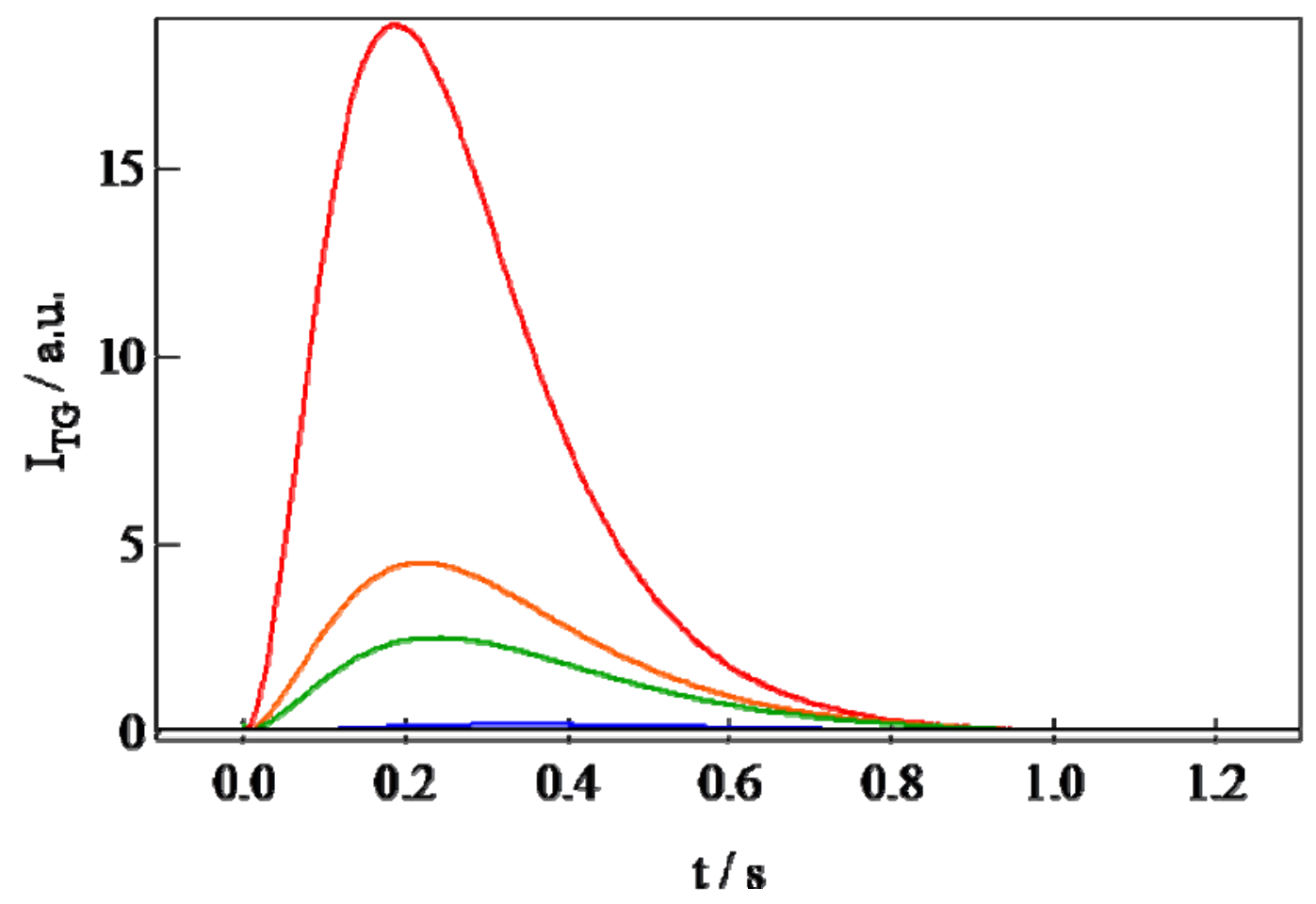

Fig.5 


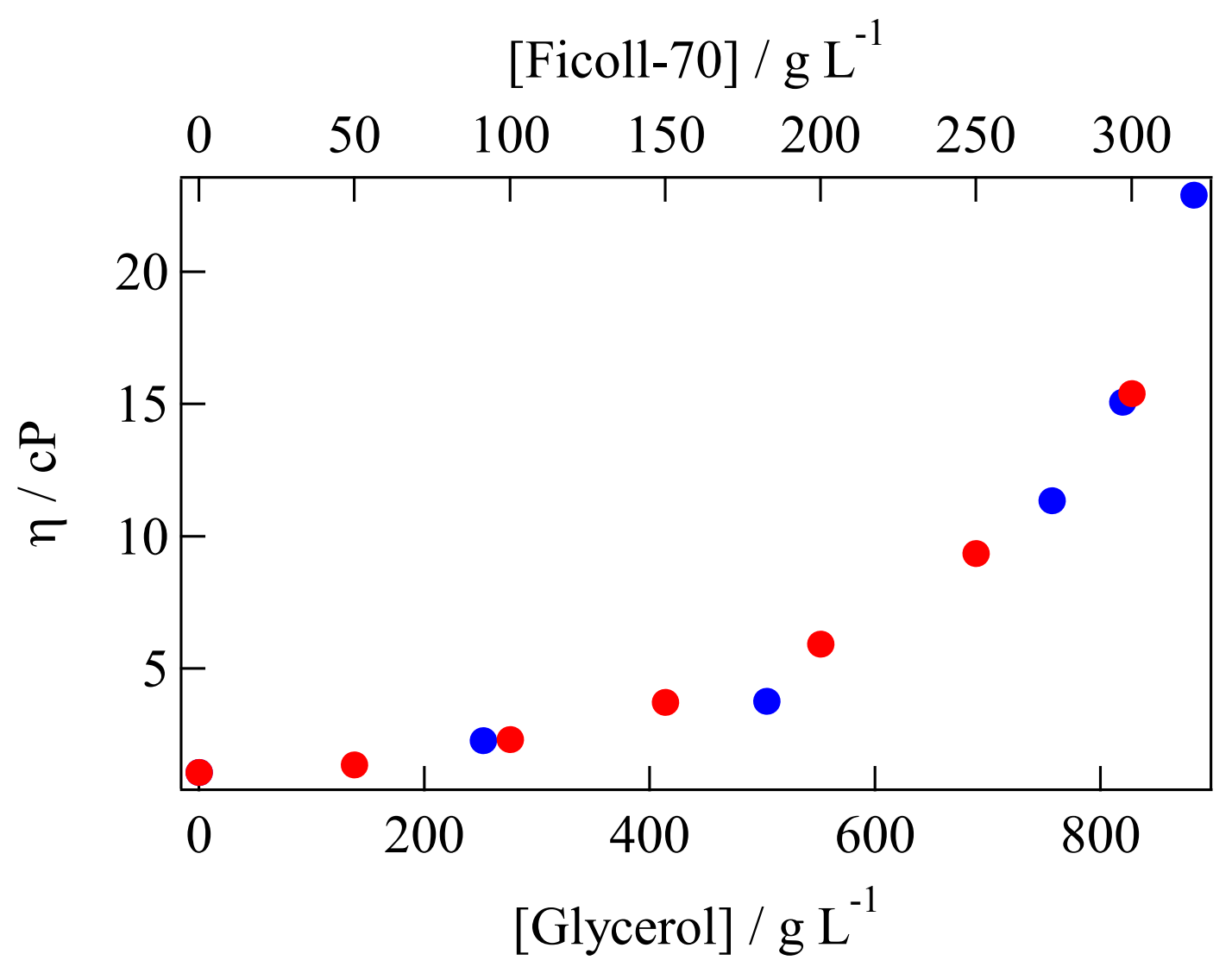

Fig.6 